\title{
An Exploration of Gender Differences through Electronic Children's Book
}

\author{
Chih-Ping Chen, Ming-Chieh Hsu \\ Yuan Ze University, Taiwan
}

\begin{abstract}
With the proliferation of online games and $e$ books all over the world, understanding users' intention to play online games through e-book learning has become a new issue for academics and practitioners. Prior studies have investigated the factors affecting learning behavioral intention to play online games. However, little research has been conducted to examine what the gender differences in playing and learning English game-based electronic children's books are. Thus, the aim of this study is to investigate gender differences in the relationships between perceived interactivity, prominence, and congruency related to game-based learning through an electronic children's book designed by our research team. Thirty-two 8-year-old (17 boys and 15 girls) Taiwanese children completed the experiments and questionnaires. Surprisingly, gender differences were predominantly not found in the relationship between factors.
\end{abstract}

\section{Introduction}

The impact of computers on our daily lives over the past few years has increased dramatically. For many of us, interacting with a computer has become a daily experience. From shopping, to entertainment, and even learning, the personal computer is fast becoming our first resource of choice. Nowadays, perhaps the most popular activity on the computer is online gaming. Indeed, Taiwan's gaming industry was estimated to be worth US\$1.4 billion (NT\$42.2 billion) in 2010, a 20\% increase from 2009, when it reached US\$1.1 billion (NT\$35.4 billion), according to figures from the Taiwan government's Industrial Development Bureau. It is also projected that the worldwide game market will be worth $\$ 100$ billion by 2015; exhibiting a $20 \%$ growth rate. Most of this growth will be seen in Asia followed by Europe and America. Furthermore, Digital Media Across Asia in 2009 highlights that the online gaming market in Taiwan is expanding and thriving, and is expected to hit record numbers of sales as each year passes. Data have revealed that the development of online games has the potential to show even more dramatic expansion in the media, entertainment, and education market in Taiwan. In other words, the development of game-based learning through e-books in Taiwan is definitely worth noting.

Gender differences are often a major concern for researchers interested in students' abilities and attitudes toward the computer or game-based learning. In the early 1980s, studies in Western countries found a large difference in the degree to which boys and girls were attracted to the computer. Beginning in the kindergarten, boys indicated more positive attitudes toward computer technology than girls did. These small attitudinal differences became dramatic in the fifth grade and continued to grow through the middle- and high-school years [15]. Furthermore, almost half of all Americans with ages 6 or older play video games [4]. Despite their popularity, a demographic rift continues to characterize the video game audience, with more men playing video games than women. Theoretically, boys are often characterized as enthusiastic users of new technology. Perhaps, it is not surprising that some commentators have speculated that it may be possible to affect positively boys' performances by supporting them through computer-aided learning tools. We argue that this is a bold claim, given that we know relatively little about how boys engage with the kinds of resources that might support their performances. It is therefore important that we do not use the notion that boys' perceived positive disposition toward computer technology as a "given" situation.

Indeed, recently there have been a number of commentators who have suggested that the gendered digital divide may be diminishing, or even disappearing [13]. Some research has shown modest improvements in the gender gap. For example, Colley and Comber [7] found that girls' interest in computer applications has improved. However, girls continue to like the computer less than boys do, and still use them less outside of school. The ESA reported that women make up 39\% of video game players, but sizable gender differences have been found in studies of children's video game play habits [4]. We argue that the results of the above studies were all based on the research of Western countries. What happened to Taiwan recently?

In addition, educators are increasingly recognizing the impact of entertainment software and utilizing games as a teaching device in a growing number of classroom settings. In doing so, they are embracing the cultural and technological shifts of the 
$21^{\text {st }}$ century and expanding the use of favorite leisure activities. E-books have combined with online games, forming into a critical and still-emerging educational resource. In fact, there has been an explosive growth of online game players over the past few decades. Statistics found that Internet users visit game-playing sites more and often stay there longer than any other Internet sites [1]; thus, playing online games has become one of the most popular learning/entertainment activities on the Internet. Bonk and Dennen [5] suggested that the massive multiplayer online games are an advanced distributed-learning technology that can provide unique education, training, and performance support opportunities. In general, game-based learning has been studied as an important alterative or supplement to traditional teaching. Many researchers have argued that perceived interactivity, prominence, and congruency have significant influences on the behavioral intention to use online games [12]. However, little research has been conducted to examine the gender differences in perception of online games in Taiwan, specifically game-based learning through e-books. Thus, the main purpose of this paper is to empirically explore the gender differences in perceived interactivity, prominence, and congruency by using a game-based English electronic children's book created by our research team for examining third grade elementary students in Taiwan. In other words, this study investigates gender differences in factors which may influence children's learning intentions, and examine these as possible contributors to differences in learning by game-based electronic children's books. This empirical study will be useful to researchers in developing and examining theories related to online games and e-books, as well as to practitioners of understanding the strategies for designing and promoting game-based learning through e-books.

The rest of this paper is organized as follows. In section 2, we establish the theoretical foundation for the research model. It is followed by the description of the experiment and data collection methods used in this study. Next, we present the hypotheses testing results. Finally, the theoretical and practical implications for future research are discussed.

\section{Theoretical Framework}

With the proliferation of online games and ebooks, understanding children's behaviors and responses to learn e-books combined with online games is becoming a hot topic in the fields of educational technology and its practitioners [2] [3]. The current study is to investigate the direct and/or indirect effects of its potential antecedents on children's behavioral intention to play online game or to learn from e-books. Integrating previous literature on education and online advertising games [6] [12] [15], this study identifies three main variables: perceived interactivity, prominence, and congruity. We developed and tested a theoretical model (Figure 1) proposing relationships between perceived interactivity, prominence, congruency and learning behavioral intention. In addition, gender difference was hypothesized to affect children's perception of game-based e-book learning and moderate the relationship between the constructs in the research model. This section elaborates on theory basis and hypotheses derivation.

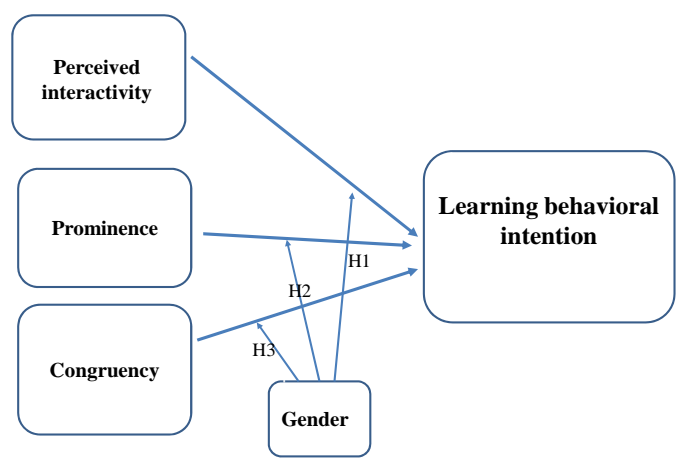

Figure 1. A Theoretical Model

\subsection{Perceived interactivity}

Interactivity is an essential feature of a successful game-based learning or e-learning [14]. One of the powers of interactivity in a game-based environment is its capability to provide efficient and appropriate interaction and feedback to the students. It has been argued that female students were less likely to take advantage of computer learning opportunities than males. Gender biases and societal stereotypes, as well as differences of interests, experiences, and attitudes, may contribute to a lower level of participation by females. It was also indicated that the concerns about gender equity expressed by many educational practitioners were well founded; female students in various levels of education tended to know less about IT, enjoy using the computer less than males, and perceive more problems with software (including interactivity with computers). In sum, the findings in previous studies showed that gender was a factor with a substantial influence on student's achievement, behavioral intentions, and usage in technology-based learning environments.

The gap in Internet usage might exist between males and females, but this gap may be the product of socioeconomic and other factors, not of gender itself. The reasons that females were less intensive Internet users might involve stereotyping, inherently "gendered" technology embodying male values, content that favored males, sex differences in cognition or communication, or other factors - in addition to socioeconomic factors.

In early days, diverse users and recipients of interactive media have differently defined the term 
"interactivity". For example: Rice [17] described it as the interaction between man and machine, and that the speed, frequency, form, and content of interaction can be controlled. Steuer [19] considered it is the interaction between messages and users, allowing immediate modification in forms and contents of messages. So far, many researchers still propose different interpretations on interactivity, yet there are studies revealing the difference between the actual level of interactivity provided by websites and the degree of perceived interactivity recognized by users [12].

Hsu and $\mathrm{Lu}$ [12] provided empirical evidence supporting that perceived interactivity is a significant determinant of online game continuance behavior. Also, some researchers have investigated if females and males maintain the same perception of all types of computer use. Scott and Rockwell [18] have shown that males reported to love playing online games or interact with others via online games more than females. Thus, it is expected that men have higher perceived interactivity and intention to play than women. This study also suggests that the influence of perceived interactivity on learning behavior intention will be moderated by gender, such that the effect will be stronger for boys. Therefore, this study examined the following $\mathrm{H} 1$ hypothesis:

\section{Hypothesis 1: Perceived interactivity positively influences behavioral intention to learn a game- based English electronic children's book more strongly for boys than girls.}

\subsection{Prominence}

Prominence used to be educators' primary consideration in teaching; it is usually the size of topics (i.e., characters, pictures, icons, and figures, etc.) for learners to remember. The topics may be identified as prominently placed or subtly placed with respect to its size and visibility. Gupta and Lord [11] defined: Prominent placements are those in which the topic is made highly visible by virtue of size or position...Subtle placements are those in which the topic is not shown prominently such as small in size.

There are different views regarding the prominent and subtle placements in advertisements and education materials. Gupta and Lord's research in movie brand placements showed that prominent brands resulted in higher recall than subtle placements. Several researchers also insist on the important role of brand prominence. For example, Matthes and his collegues' study of brand placements in television programs demonstrated that prominent brand placement resulted in high recall, but had negative brand attitudes for viewers who showed awareness of the deliberate brand placement and low involvement with the program. A prominent brand placement is more deeply processed and that leads to increased memory.

Indeed, large topics often attract attention and enhance recall more effectively than small ones. In education, the position of a topic is also important because the learner may not realize the existence of the topic in poor positions, especially topics installed in online games or online advertising games. In such games, the learners' main focus may be laid outside the area of the topic, in which they could not recall or identify the messages conveyed by the topics [1]. In a gaming environment, it was found that placing topics in the foreground (prominent) of the game increased its recall [11]. Furthermore, some Taiwanese studies have shown that males recall more information about the foreground of the online games than females do. Therefore, we expect that boys are more influenced in a high prominence game-based electronic children's book and have a higher intention to learn than girls. Thus, the following hypothesis was tested:

\section{Hypothesis 2: Prominence on a game-based electronic children's book positively influences behavioral intention to learn English more strongly for boys than girls.}

\subsection{Congruency}

Congruency in different research approaches has been widely used in studies on education, psychology, and online game advertising [9]. There were relevant studies such as the congruency between advertising and the presentation of ad contents, and the congruency between products and their brand names [16]. Luna and Peracchio [14] conducted a study with Spanish/English speakers. Their findings revealed that evaluation of web sites containing information presented in a second language was enhanced by congruent graphics with the verbal content, whereas evaluation of web sites containing information presented in the first language was favored when low congruency existed. In particular, in the electronic game context, topic placements that match real life concepts enhance the playing experience. Similarly, electronic game players enjoy topic placement displays when they make sense in the game context.

A limited body of research addresses the effect of congruency or connection between entertainment content and an embedded subject in placement. In fact, the proportion of children with access to online games and e-books is increasing. Their trial age is getting younger, and these devices are becoming a bigger part of young children's lives at home and at school. Some companies provide online games related to a major concept with its brand to gain children's attention. The congruency of contents is being created based 
on children's interests, which are becoming popular tools for companies or educators to connect with their concepts. An example is the Barbie website, which describes itself as an "online game for girls," and has attracted one million visitors each month. At the site, children are encouraged, to spend time in a branded environment in which all the content directly or exclusively related to Barbie products through a wide selection of online games. Another congruity example is when Kellogg's provided an online game called Froot Loops in early 2005. More importantly, this online game shows a preference for Froot Loops over fresh fruit by rewarding the child 10 points for successfully throwing a Froot Loop into the monster's mouth, while a piece of fruit (e.g., peach or apple) earns only 5 points. Furthermore, the monster makes a moderate "mmm" sound when it eats fresh fruits, while a very loud and more satisfying "mmmmmm” sound after a Froot Loop is successfully thrown into its mouth. The game's encouragement to prefer Froot Loops over fruit is particularly questionable because Froot Loops cereal has the highest portion of processed sugar of major cereals sold in Perth, and should be consumed in moderation. Moreover, the studies in Taiwan found that girls more cared about the congruency between game and learning contents than boys. This study further contends that girls will have higher congruency between game and learning contents than boys. Thus, this study tested the following hypothesis:

Hypothesis 3: The congruency between the game and learning contents positively influences learning behavioral intention to play an electronic children's book more strongly for girls than boys.

\section{Methods}

\subsection{Experimental stimuli}

English is important for third grade elementary students in Taiwan to start learning foreign language. It also becomes a critical issue for educators to understand how to attract children's attention and their learning behavioral intention. Since online games genre simulates real life for elementary students, it is expected that combined English learning with online games extend to the electronic children's book setting. Hence, a game-based English learning through an electronic children's book was created for this study based on three factors (i.e., perceived interactivity, prominence, and congruency) (See Figure 2, 3, 4). To test the stated hypotheses, the experiment was conducted in the laboratory in Taoyuan elementary school in Taiwan. The sample population consisted of children with the age of nine who were gaining their initial independent experiences using the Web and e-book devices. Children in this age can respond to simple paper and pencil surveys. These were the skills required for the experiment. A total of 32 third grade elementary students including 17 boys and 15 girls participated in the experiment.

\subsection{Measure}

The final questionnaire consisted of twelve questions, each displaying multiple color drawings as possible answers. The survey and researcher asked the children to circle only one option per question. Pretests with participants in the target age were conducted to confirm the suitability of the game-based English electronic children's book used as a treatment stimulus, the data collection venue, procedures, and measures undertaken.

\subsection{Experimental Procedure}

The experiment was conducted by two female researchers and supervised by a teacher or teacher's assistant when she was in contact with the participants. As noted earlier, this experiment was conducted in computer lab of Taoyuan elementary school in Taiwan. Children participated in the experiment at a certain time. After each child had played the game-based English electronic children's book at least twice (about fifteen minutes of play), the questionnaire was provided. It was in the form of a booklet that had several questions per page, with their possible answers (drawings or photographs) printed in color.

Figure 2. A Game-based English learning e-book created different buttons by interactivity

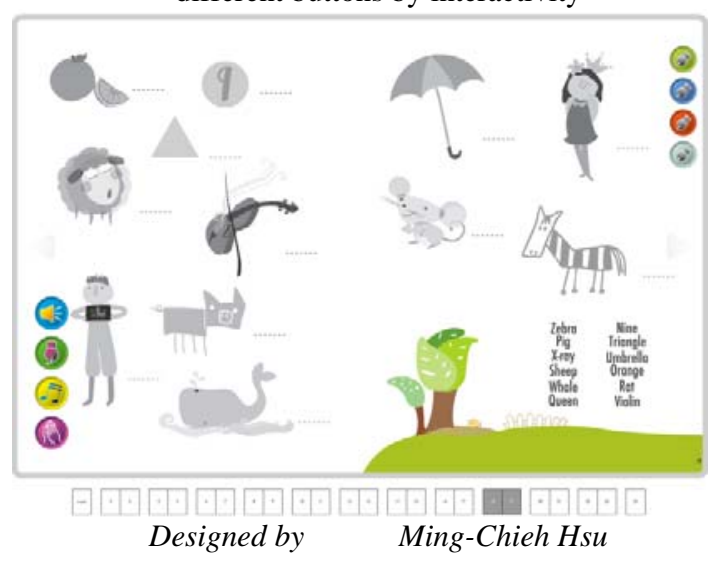

Figure 3 A Game-based English learning e-book created by prominent $\&$ subtle placement

The answers were placed in several positions relative to one another to account for any potential effect in the position order effects that were found. The researchers proceeded through aiding the children with the questionnaire, reading out each question and using A4 paper - sized examples of 
the possible answers for visual aids. The experiment and data collection from the children averaged approximately 20 minutes. Debriefing the children after collecting their responses failed to find any children who were aware of the objectives of the research, or the hypotheses. In other words, potential demand effects in the children's responses were not apparent from this test, but can never be ruled out with young respondents.

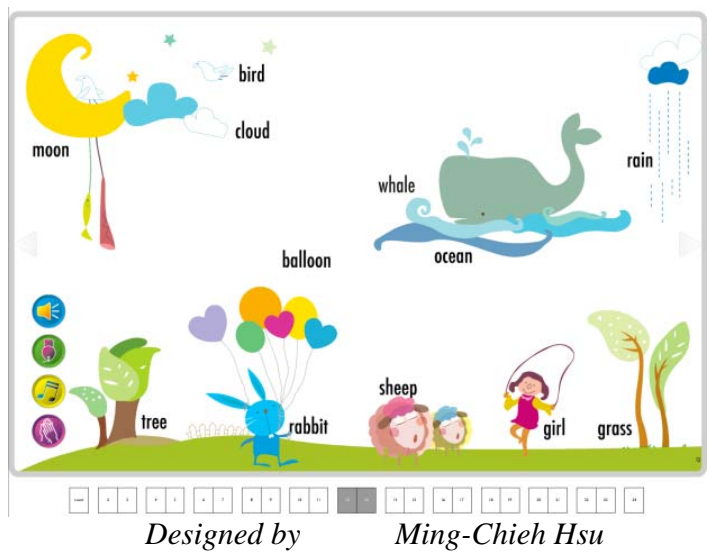

Figure 4 A Game-based English learning e-book created by congruency

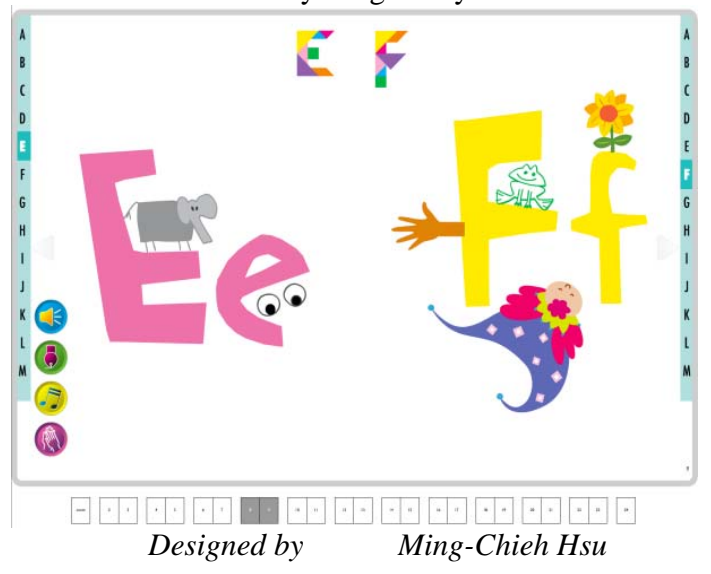

4. Results

\subsection{Assessment of perceived interactivity}

The children in the experiment were provided with a game-based English electronic children's book created by our research team. This provided a scale from 1 to 5 choices. Hypothesis 1 proposes that boys who played and learned from a game-based English electronic children's book will tend to like perceived interactivity positively influences behavioral intention than girls. In this study, there were no significant differences $(t=-.651, p=.520)$ between perceived interactivity and learning behavioral intention for gender (boys $M=4.3235$ versus girls $M=4.4778$ ) on this measure (See Table 1). There is no support for the hypothesis that perceived interactivity positively influences behavioral intention to learn a game-based English electronic children's book more strongly for boys than girls.

\subsection{Prominence for boys and girls}

The second hypothesis proposes that prominence on a game-based English electronic children's book positively influences behavioral intention to learn more strongly for boys than girls. As Table 1 shows, there were no significant differences $(t=.898 ; p=$ $.376)$ between boys $(M=4.2118)$ and girls $(M=$ $4.0533)$ to recall or prefer prominence on a gamebased English electronic children's book. In other words, there is also no support for hypothesis 2 that having prominence on game-based English learning through an electroninc children's book results to different preferences between genders.

\subsection{Congruency for gender}

Hypothesis 3 examines that a significantly higher proportion of girls would report that they intend to learn and prefer the congruency between game and learning contents than boys. As Table 1 reveals, the difference in proportions obviously wasn't significant $(t=-.515 ; p=.610)$. There was no support that girls $(M=4.2444)$ strongly want to learn and prefer the congruency between game and learning contents, compared with boys $(M=4.1569)$.

Table 1. Gender differences on game-based English learning through electronic children's book

\begin{tabular}{lllllll}
\hline Variances & Groups & $\#$ & Mean & ST & $\boldsymbol{t}$ & $\boldsymbol{p}$ \\
\hline Perceived & Boys & 17 & 4.3235 & .74877 & & \\
interactivity & Girls & 15 & 4.4778 & .56297 & -.651 & .520 \\
\hline \multirow{2}{*}{ Prominence } & Boys & 17 & 4.2118 & .54987 & & \\
& Girls & 15 & 4.0533 & .43072 & .898 & .376 \\
\hline \multirow{2}{*}{ Congruity } & Boys & 17 & 4.1569 & .48042 & & \\
& Girls & 15 & 4.2444 & .47920 & -.515 & .610 \\
\hline
\end{tabular}

\section{Discussion and Conclusion}

This study provided initial insight into gender learning on game-based learning through an electronic children's book, especially focused on three theoretical factors such as perceived interactivity, prominence, and congruency. Although children who played a game-based English electronic children's book reported a significantly higher preference for our designed e-book, there were no differences by gender in their perceived interactivity with the e-book. This finding is different from previous Taiwanese studies that examined college students where perceived interactivity positively influenced the behavioral intention to play online games more strongly for college male students than for females. In prominence on game-based English learning through an electronic children's book, there are no studies to compare within this 
issue by gender differences. The effect of prominence on gaming intention, however, had no influence on preferences of different gender on children's learning. Unsurprisingly, there were no differences by gender in the congruency between game and learning contents to positively influence behavioral intention to play e-book. In other words, the results of this study did not reveal any significant differences between boys and girls on their learning behavioral intentions.

It suggested that they had similar attitudes toward the proliferation and development of online games and e-book technology as a learning tool to reduce the digital divide and gender stereotypes. This result was noteworthy because it contradicted the findings of some studies regarding the relationship between technology and gender - suggesting that computing is a masculine-dominated technology, that gendering of computer (Internet) technologies exist, and characterizing females' relationship to the computer as problematic or disadvantaged. This study found that boys and girls held statistically similar attitudes on the dimension of access to game-based English electronic children's book for learning.

In sum, these simple findings clearly suggest gender differences is getting vague between boys and girls in learning from game-based English electronic children's books in Taiwan. An overview of research in the past few decades draws to the conclusion that females are at a disadvantage relative to men when learning about computers or learning other material with the aid of computer-assisted software in Taiwan. However, with economic and academic success depending more and more on a facility with information technology, this study shows that any disparity in interest or proficiency is becoming to bridge the gap by breaking the gender stereotype in Taiwan. Cooper and Weaver [8] speculated that "gender stereotypes can have the power of the selffulfilling prophecy, creating further evidence for the stereotype" (p. 328). He further concluded that the "mere knowledge of a negative stereotype applying to a person's group can cause that person to perform poorly at a particular task” (p. 329). Based on the concept of Cooper and Weaver, we also suggest that educators in Taiwan need to make efforts not to create gender stereotypic environments for children. Once children get used gender-unbiased environments, they perform naturally.

\section{Limitations and Further Research}

Given the limitations of the current study, there are opportunities for further research. The hypotheses of the current study developed by applying theoretical framework were largely developed in traditional advertising media and product placement to interactive media. We argue that applying traditional advertising mechanisms to a new interactive media environment and crossing to different fields (i.e., educational learning field) should be done with care. Further research should focus more in depth on mechanisms such as persuasion knowledge in a game-based learning environment. Although the convenience samples used were appropriate to reach relevant samples of learners, it might have decreased the external validity of the study. Further research that uses different sampling methods is needed to validate these results.

\section{References}

[1]. A. Acar, "Testing the effects of incidental advertising exposure in online gaming environments", Journal of Interactive Advertising, (2007). 8(1), 1-36.

[2]. R. Anni Layne, "More than a game”, Fast Company, (2002). (58), 46.

[3]. K. T. Anuradha, and H. S. Usha, "Use of an e-book in an academic and research environment", Electronic Library and Information Systems, (2006), 40, 48-62.

[4] E. S. Association, "Game player data", (2005), Available http://www.theesa.com/facts/gamer_data.php. (Access date: 2 August 2012).

[5] C. J. Bonk, \& V. P. Dennen, Massive multiplayer online gaming: a research framework for military training and education (Technical Report 2005-01). (2005), Washingto, DC: Office of the Under Secretaty of Defense for Personnel and Readiness.

[6] V. Cauberghe, “Advergames”, Journal of Advertising, (2010), 39(1), 5-18.

[7] A. Colley, \& C. Comber, “Age and gender differences in computer use and attitudes among secondary school students: what has changed?", Education Research, (2003), 45, 155-165.

[8] J. Cooper, \& K. D. Weaver, Gender and computers: Understanding the digital divide. (2003), Mahwah, NJ: Lawrence Erlbaum Associates.

[9] R. J. Faber, \& M. Lee, "Effects of product placement in online games on brand memory: A perspective of the limited-capacity model of attention”, Journal of Advertising, (2007), 36(4).

[10] J. B. Funk, \& D. D. Buchman, “Children's perceptions of gender differences in social approval for playing electronic games”, Sex Roles: A Journal of Research, (1996), 35, 219-231.

[11] P.B. Gupta and K.R. Lord, "Product placement in movies: The effect of prominence and mode on audience recall”, Journal of Current Issues and Research in Advertising, (1998), 20(1), 47-59.

[12] C. Heeter, "Interactivity in the context of designed expreiences”, Journal of Interactive Advertising, (2000), 1(1), 53-62. 
[13] S. C. Losh, "Gender, educational, and occupational digital gaps -- 1983-2002”, Social Science Computer Review, (2004), 22, 152-166.

[14] D. Luna and L.A. Peracchio, "Bilinguals, Graphics, and the Web: A Language Processing Approach", Advances in Consumer Research, (2002). 29, 186-187.

[15] V. Mallinckrodt, \& D. Mizerski, "The effects of playing an advergame on young children's perceptions, preferences, and requests”, Journal of Advertising, (2007), 36(2), 87-100.

[16] J. Matthes, C. Schemer, and W. Wirth, "More than meets the eye: investigating the hidden impact of brand placements in television magazines", International Journal of Advertising, (2007). 26(4), 447-503.

[17] R. E. Rice, Development of new media research. In R. E. Rice (Ed.), The New Media: Communication, Research, and Technology (pp. 15-31). (1984), Beverly Hills, CA: Sage.

[18] C. R. Scott, \& S. C. Rockwell, "The effect of communication, writing, and technology apprehension on likelihood to use new communication technologies", Communication Eucation, (1997), 46(1), 44-62.

[19] J. Steuer, "Defining virtual reality: Dimensions determining telepresence", Journal of Communication, (1992), 42(4), 73-93. 